\title{
Labyrinthe
}

15 | 2003

Territoires : questions d'images

\section{Karin Weingartz-Perschel, Der Holocaust als Paradigma Instrumenteller Verwertungslogik, Francfort, Peter Lang, 2002}

\section{Laurent Ferri}

\section{(2) OpenEdition \\ Journals \\ Édition électronique \\ URL : http://journals.openedition.org/labyrinthe/482 \\ DOI : $10.4000 /$ labyrinthe.482 \\ ISSN : 1950-6031 \\ Éditeur \\ Hermann}

Édition imprimée

Date de publication : 1 juin 2003

\section{Référence électronique}

Laurent Ferri, « Karin Weingartz-Perschel, Der Holocaust als Paradigma Instrumenteller Verwertungslogik, Francfort, Peter Lang, 2002 », Labyrinthe [En ligne], 15 | 2003, mis en ligne le 24 juillet 2008, consulté le 28 avril 2019. URL : http://journals.openedition.org/labyrinthe/482 ; DOI : 10.4000/labyrinthe.482

Ce document a été généré automatiquement le 28 avril 2019

Propriété intellectuelle 


\title{
Karin Weingartz-Perschel, Der Holocaust als Paradigma Instrumenteller Verwertungslogik, Francfort, Peter Lang, 2002
}

\author{
Laurent Ferri
}

Le citoyen vertueux membre de la société allemande contemporaine se doit d'apprendre à regarder en face son douloureux passé afin de tirer les leçons de la barbarie nazie. Dans la réalisation de cette noble tâche, l'expert tiendrait le rôle d'éclaireur avisé. Et justement, de manière très symptomatique, Karin Weingartz-Perschel, chercheuse en anthropologie et psychologie sociales ainsi qu'en Women- et gender studies à l'université de Düsseldorf, repose la question du génocide nazi, non pas du point de vue de ses rouages et de sa chronologie, mais de celui de ses racines idéologiques et scientistes. On ne peut pas dire que son ouvrage manque d'ambition : «On a beaucoup écrit sur le thème de l'Holocauste, d'Auschwitz, du national-socialisme, du nazisme [...]. Et pourtant, nous ne disposons aujourd'hui que d'une réponse partielle à la question du Pourquoi (quatrième de couverture et p. 7).»

2 Le ton se veut dramatique : la guerre entre les faibles et les forts, entre les dominants et les dominés, n'aurait pas atteint son zénith pendant la période nazie, en dépit «des cruautés inouïes et [du] perfectionnement bureaucratique » de celle-ci. Mais nous serions actuellement "en plein dans cette guerre ", et pour la première fois, approcherions de « la maîtrise de techniques génétiques qui, sans effusion de sang et de manière invisible, nous permettent de nous débarrasser des existences que nous traînons comme des boulets, du moins d'empêcher qu'elles adviennent » (p. 8). La Shoah, apprend-on, n'aurait été que la première mise en pratique d'une obsession sociale-darwinienne du surhomme (il est question d'une curieuse «tradition nitzschéo-darwinienne » p. 33). Cette dernière aurait ainsi coïncidé non seulement avec l'idéologie bourgeoise de la normalité, mais aussi avec les intérêts d'un capitalisme consacrant la loi du plus fort, du plus normal, et 
condamnant les improductifs et les anormaux, autrement dit tous ceux qui ne produisent pas de valeur économique (die Unwerten ou "sans-valeur»). Sous le III ${ }^{e}$ Reich comme aujourd'hui, une telle coïncidence ne peut que dégénérer en "guerre de tous contre chacun » (p. 132). L'« actualité » de l'Extermination résiderait donc dans les tentations et les moyens actuels $d u$ « biopolitique ${ }^{1}$ » défini par Michel Foucault). On ne le sait guère en France, mais le Parc humain de Peter Sloterdijk ${ }^{2}$ fit débat en Allemagne il y a quatre ans; presque autant que l'historien Nolte en 1986, à l'occasion de la fameuse "querelle des historiens » sur le nazisme. Il y a peu de chance que le livre de Weingarzt-Perschel connaisse le même écho.

3 En effet, nous n'y trouvons rien de très original : le lecteur y reconnaîtra, radicalisées et recyclées, les analyses et les réflexions issues de l'école de Francfort, tendance MarcuseReich. Typique est la satire des structures familiales- bourgeoises les plus régressives: psychologie de la soumission, respect des hiérarchies reconduites, fantasme sadomasochiste de la race comme extension de la famille... Nous lisons ainsi que «le fascisme est tout le contraire d'une irruption brutale dans la société bourgeoise et ses institutions : c'est la consécration de leur normativité » (p. 112).

Seulement, depuis trente ans, les travaux des historiens permettent tout de même une approche moins unicausale des années 1933-1945. Pour commencer, il n'est guère acceptable d'étudier l'Holocauste en dehors de toute périodisation, et de considérer le nazisme comme un bloc homogène et linéaire. Que Auschwitz accomplisse Mein Kampf n'est pas discutable. Mais nous savons que l'extermination physique des Juifs d'Europe, et d'autres catégories d'Untermenschen ne fut accomplie qu'au terme d'un processus complexe. Elle ne fut qu'une des options finalement choisies. Laborieuse fut la définition même du «Juif», tout comme celle de l'«inadapté social» - terminologie la plus extensive, ouvrant à la «dialectique du meurtre» (Hannah Arendt) des perspectives infinies, y compris l'élimination interne des anciens bourreaux par de nouveaux. Les récentes et passionnantes études de Raoul Hilberg (Sources of Holocaust Research, 2001) sont encore venues enrichir une bibliographie qui fait, hélas! défaut à notre polygraphe.

5 La stigmatisation incessante de «la bourgeoisie complice du nazisme » laisse également sceptique. Il est vraiment simpliste d'évacuer la problématique des appuis sociaux d'Hitler en accablant seulement le grand capital sur la base de quelques «indices » fragiles (par exemple le fait que Krupp ait remis un prix à un médecin eugéniste en 1900). Jusqu'en 1933, le dictateur trouva la masse de ses supporters dans la classe ouvrière, la paysannerie et la petite bourgeoisie déclassée. Cette contradiction souvent relevée, d'un mouvement élitiste s'appuyant sur les perdants de la société, ne pouvait trouver sa résolution que dans une exaltation de la valeur raciale et un renversement des critères hiérarchiques, assortis d'une amélioration des conditions de vie : la seule élimination des ennemis raciaux n'aurait pas suffi à mobiliser $90 \%$ d'une population. Comme l'a montré Visconti dans Les Damnés, les grandes familles se rallièrent aux racistes en majorité, mais par opportunisme, et sans illusion durable : dès 1936, on nota un effacement des élites économiques, tout simplement parce que le capitalisme ne peut être entièrement orienté, sous contrôle d'une bureaucratie d'État, vers une guerre de conquête et d'extermination. Quand l'auteur écrit : «Il n'y avait qu'un seul but : l'avènement du peuple aryen comme race des seigneurs» (p. 53), on ne voit pas le lien avec le culte «bourgeois» du travail. Certes, l'élimination des handicapés relève d'une logique productiviste ; mais enfin aucun déporté ne fut jamais libéré pour rendement exceptionnel, malgré la devise Arbeit macht frei qui ornait les grilles de Buchenwald. Certes, l'IG-Farben, principal trust industriel, fut 
complice de l'Holocauste : mais, comme s'en plaignaient les ingénieurs, il est un moment où la surexploitation et l'élimination par le travail entrent en contradiction, comme le voit l'auteur elle-même: «L'avènement d'une race germanique nécessitait la mise en place le plus rapidement possible d'une industrie de l'élimination de tous les parasites infestant le corps du peuple; en même temps, l'anéantissement de ceux qui, parmi ces prétendus parasites, pouvaient travailler, contredisait toute logique économique à un moment où le besoin de main-d'œuvre valable se faisait croissant» (p. 85). La récupération des corps n'a jamais fourni que des sources de revenus médiocres. Et quand les trains et les militaires continuaient, en 1944, d'être réquisitionnés pour acheminer des déportés, contre toute "rationalité », il est difficile de plaider la victoire de la pensée technicienne. Du reste, les représentants des élites bourgeoises ralliées (et il ne faudrait ici oublier ni l'exil de la famille Mann ni le repentir d'un Thyssen) ne croyaient d'ailleurs plus en Hitler.

Mais peut-être nous méprenons-nous sur les intentions et, in fine, sur le statut du livre de Weingartz-Perschel : il n'est qu'une machine de guerre contre l'éthique conservatrice et contre l'économie de marché. La postface est instructive, qui oppose la figure de Berlusconi (nouvel Hitler ?) aux antimondialisation (?). Il s'agit, dès lors, d'éviter tout questionnement qui pourrait gêner la formation du front antiglobalisation, par exemple : la survalorisation du capital (démographico-génétique, industrialo-financier) fut-il le seul fondement $\mathrm{du}$ tri des individus au $\mathrm{xx}^{\mathrm{e}}$ siècle ? Le communisme soviétique, à la fois synonyme de productivisme et de gaspillage, a théorisé puis pratiqué le génocide selon d'autres grilles, révolutionnaire ou nationale-sociale (élimination des koulaks en tant que classe, déportation d'ethnies "contre-révolutionnaires»). Cela ne doit-il pas nous détourner de tout délire antiéconomique, et de toute pensée du complot scientiste, même revêtus des prestiges de l'autorité (pseudo) intellectuelle et camouflés sous les oripeaux d'une « réforme » (p. 157) aux contours bien imprécis?

\section{NOTES}

1. Le «biopolitique » renvoie à ce qui est jugé légitimement supprimable, car ne méritant pas de vivre.

2. Théorisé par Peter Sloterdijk, le "parc humain" est la sphère de domestication et de "planification explicite des êtres humains", dont les corps font incessamment l'objet de manipulations et d'échanges technico-génétiques, et les esprits l'objet de manipulations massmédiatiques. Le "fascisme d'amusement " traduirait alors un "retour à l'état sauvage ». Voir Règles pour le parc humain [1999], traduit de l'allemand par Olivier Manoni, Paris, Les Mille et une nuits, 2000. 